\title{
Selection of Plasmodium falciparum pfcrt and pfmdr1 polymorphisms after treatment with artesunate-amodiaquine fixed dose combination or artemether-lumefantrine in Liberia
}

Sabina Dahlström Otienoburu ${ }^{1,2,3^{*}} \mathbb{D}$, Oumou Maïga-Ascofaré ${ }^{1,4}$, Birgit Schramm ${ }^{5}$, Vincent Jullien ${ }^{6}$, Joel J. Jones ${ }^{7}$, Yah M. Zolia ${ }^{7}$, Pascal Houzé ${ }^{8}$, Elizabeth A. Ashley ${ }^{5,9}$, Jean-René Kiechel ${ }^{10}$, Philippe J. Guérin ${ }^{2,5,9}$, Jacques Le Bras ${ }^{1,11,12}$ and Sandrine Houzé ${ }^{11,12}$

\begin{abstract}
Background: Plasmodium falciparum uncomplicated malaria can successfully be treated with an artemisinin-based combination therapy (ACT). However resistance is spreading to the different ACT compounds; the artemisinin derivative and the partner drug. Studies of $P$. falciparum polymorphisms associated with drug resistance can provide a useful tool to track resistance and guide treatment policy as well as an in-depth understanding of the development and spread of resistance.
\end{abstract}

Methods: The role of P. falciparum molecular markers in selection of reinfections was assessed in an efficacy trial comparing artesunate-amodiaquine fixed-dose combination with artemether-lumefantrine to treat malaria in Nimba County, Liberia 2008-2009. P. falciparum polymorphisms in pfcrt 76, pfmdr1 86, 184 and 1246, and pfmrp1 876 and 1466 were analysed by PCR-RFLP and pyrosequencing.

Results: High baseline prevalence of pfmdr1 1246Y was found in Nimba county (38\%). Pfmdr1 1246Y and pfmdr1 86+184+1246 haplotypes NYY and YYY were selected in reinfections in the artesunate-amodiaquine arm and pfcrt K76, pfmdr1 N86 and pfmdr1 haplotype NFD were selected in artemether-lumefantrine reinfections. Parasites harbouring pfmdr1 1246Y could reinfect earlier after treatment with artesunate-amodiaquine and parasites carrying pfmdr1 N86 could reinfect at higher lumefantrine concentrations in patients treated with artemether-lumefantrine.

Conclusions: Although treatment is highly efficacious, selection of molecular markers in reinfections could indicate a decreased sensitivity or tolerance of parasites to the current treatments and the baseline prevalence of molecular markers should be closely monitored. Since individual drug levels and the day of reinfection were demonstrated to be key determinants for selection of reinfections, this data needs to be collected and taken into account for accurate evaluation of molecular markers for anti-malarial treatments.

The protocols for the clinical trial was registered with Current Controlled Trials, under the Identifier Number ISRCTN51688713 on 9 October 2008

Keywords: Plasmodium falciparum, Malaria, Antimalarial agents, Artemisinin-based combination therapy, Drug resistance, Selection, pfmdr1, pfcrt, pfmrp1

\footnotetext{
*Correspondence: sabina.dahlstrom@gmail.com

${ }^{1}$ Institut de Médecine et d'Epidémiologie Appliquée, Bichat-C. Bernard Hospital, Paris, France

Full list of author information is available at the end of the article
} 


\section{Background}

Plasmodium falciparum malaria is a devastating disease still causing high mortality and morbidity especially in children in sub-Saharan Africa. The uncomplicated form of $P$. falciparum infection can be easily and successfully treated with an artemisinin-based combination therapy (ACT); however there is always the threat of resistance development to the different ACT compounds; the artemisinin derivative and/or the partner drug. Studies of $P$. falciparum polymorphisms associated with drug resistance can provide a useful tool to track resistance and guide treatment policy as well as an in-depth understanding of the development and spread of resistance.

Polymorphisms in $p f m d r 1$ and $p f c r t$ have been shown to have an effect on parasite susceptibility to artesunate-amodiaquine (AS-AQ) treatment, in particular pfcrt 76T, pfmdr1 1246Y and the pfmdr1 86-184-1246 haplotype $\mathrm{Y}-\mathrm{Y}-\mathrm{Y}$ which has been associated with recrudescences and reinfections [1-4]. Pfmdr1 N86, 184F and D1246 and pfcrt K76 alleles are repeatedly demonstrated to be selected in reinfections or recurrent infections after artemether-lumefantrine (AL) treatment $[5,6]$, supporting their role in the decreased sensitivity to lumefantrine. In pooled analyses, an increased risk of recrudescence after AL treatment was demonstrated when pfmdr1 N86 was present [4].

Only scarce data on anti-malarial efficacy and prevalence of molecular resistance markers is available from Liberia. In 1978-1981, wildtype alleles pfcrt K76 and pfmdr1N86 were dominating [7]. In 2000, high clinical resistance to chloroquine was observed as well as high baseline prevalence of the chloroquine resistance marker pfcrt 76T (84 \%) [8]. AS-AQ became the first line treatment in 2003 and it was changed to AS-AQ fixed dose combination (ASAQ-FDC) in 2010. High efficacy of ASAQ-FDC and AL was demonstrated in this clinical trial conducted in 2008-2009 [9]. The aim of the study was to investigate the prevalence and selection of $p f c r t$ and pfmdr1 genotypes in the clinical trial. This is the first study to assess molecular anti-malarial resistance markers in Liberia since the implementation of ACT. The work shows selection of parasite molecular markers in reinfections after treatment with both ACT and that molecular markers can influence the time after treatment and at which drug concentration a parasite is able to reinfect.

\section{Methods}

\section{Study subjects}

Analyses were performed of $P$. falciparum positive blood samples from a randomized non-inferiority efficacy trial evaluating ASAQ-FDC $\left[\mathrm{n}=149\right.$ (ASAQ Winthrop ${ }^{\circledR}$, Sanofi-Aventis)] and AL [ $\mathrm{n}=150$ (Coartem ${ }^{\circledR}$, Novartis)], conducted in 2008-2009 in Saclepea Comprehensive
Health Centre in Nimba County, Liberia-a facility at the time supported by Médecins Sans Frontières-Switzerland in collaboration with the Ministry of Health. Children below 5 years of age were enrolled and followed for 42 days. The protocols for the clinical trial was registered with Current Controlled Trials, under the identifier number ISRCTN51688713 [9]. Blood was collected from the patients on FTA filter papers before treatment and during the follow up.

Parasite molecular markers in pfcrt K76T, pfmdr1 N86Y, Y184F, D1246Y and pfmrp1 I876V and K1466R was assessed in pre-treatment and post-treatment samples, classified according to the 42-day PCR-adjusted outcome. Recrudescences and reinfections were distinguished by stepwise genotyping $m s p 1, m s p 2$ and glurp [9].

\section{DNA extraction}

DNA was extracted from blood spotted onto FTA filter papers using the QIAamp ${ }^{\circledR} 96$ DNA Blood Kit according to the manufacturer's protocol for dried blood spots (Qiagen, Hilden, Germany).

\section{Pyrosequencing}

pfmrp1 SNPs in codons I876V and K1466R were analysed from extracted DNA by PCR amplification followed by pyrosequencing previously described [3]. The definition of a single genotype (pure) infection was a pyrosequencing result above $90 \%$ or below $10 \%$, while a mixed genotype infection was between 10 and $90 \%$ for both genotypes.

\section{Nested-PCR and RFLP}

$P f c r t$, and $p f m d r 1$ SNPs were analysed by nested PCR followed by restriction fragment length polymorphisms as previously described [3].

\section{Determination of drug concentrations in blood and serum} Pre-treatment serum samples were tested for previous intake of chloroquine and quinine by liquid chromatography coupled with tandem mass spectrometry. Pre-treatment and day 7 concentrations of desethylamodiaquine (DAQ) and lumefantrine were analysed from venous blood spotted on filter paper and measured by high performance liquid chromatography with ultraviolet detection (lumefantrine) or by tandem-mass spectrometry [4].

\section{Data analyses}

Fisher's two-tailed test (GraphPad Software Inc. Sand Diego, CA) was used to evaluate genetic selection by the difference in genotype prevalence between pretreatment samples and recrudescences and reinfections, respectively. The baseline genotype prevalence was based 
on pre-treatment samples from both treatment arms. Patients were excluded from the pre-treatment group due to incorrect enrolment and infection with other species and from the post-treatment analysis due to infection with other species, missing or undetermined result from the msp1, msp 2 and glurp genotyping or incomplete treatment. In one SNP analysis, mixed genotypes were analysed together with the non-selected genotypes against the selected genotype, as previously suggested [3]. For example, in the AL arm the prevalence of mixed alleles in position pfmdr1 $86(\mathrm{~N}+\mathrm{Y})$ was added to the prevalence of $p f m d r 186 \mathrm{Y}$ and compared to the prevalence of $p f m d r 1$ N86. Mixed genotype infections were excluded in the haplotype analyses. Mann-Whitney U test was used to assess differences in drug concentrations at day 7 and time of reinfection in relation to parasite genotype. Exclusion criteria were incomplete treatment and missing data. Statistical significance was defined as a $p$ value $\leq 0.05$ in all analyses.

\section{Results}

\section{Baseline prevalence of polymorphisms}

Before treatment the prevalence of the $p f c r t$ mutant allele $76 \mathrm{~T}$ was $93.6 \%$. There was large variation in the $p f m d r 1$ SNPs where the mutant alleles $86 \mathrm{Y}, 184 \mathrm{~F}$ and $1246 \mathrm{Y}$ were found in 69.4, 43.6 and $38.4 \%$ respectively (including mixed genotype infections). The wild type allele was predominating in pfmrp 1; I876 was found in $99.3 \%$ of the samples and K1466R in $97.6 \%$ (Table 1). Previous intake of chloroquine, quinine or amodiaquine/DAQ (measured in pre-treatment serum samples), did not have significant impact on the baseline prevalence of pfcrt and pfmdr1 SNPs.

\section{Selection of polymorphisms after treatment}

After treatment pfmdr1 $1246 \mathrm{Y}$ was selected in the ASAQ-FDC arm, which was found in 55/294 (18.7 \%) patients at baseline and in 24/60 (40.0\%) reinfections (Fisher, two-tailed, $p<0.001$ ) (Table 1 ). In the AL arm, a selection of pfcrt K76 and pfmdr1 N86 was identified, that increased in prevalence from 19/294 (6.5\%) and 90/294 (30.6\%) at baseline to $12 / 38$ [31.6\% $(p<0.0001)$ ] and $28 / 40[70.0 \%(p<0.0001)]$, respectively, in reinfections (Table 1). These results were confirmed when omitting mixed genotype infections from the analyses. When studying the pfmdr1 haplotype $86+184+1246$, NYY and YYY were selected in reinfections after ASAQ-FDC treatment, with a baseline prevalence of 0/198 $(0 \%)$ and $47 / 198(23.7 \%)$ and reinfection prevalence of $2 / 49$ [4.1\% $(p=0.04)]$ and $20 / 49$ [40.8 \% $(p=0.02)]$, respectively, while the prevalence of the wild-type haplotype NYD decreased from 27.8 to $10.2 \%(p<0.01)$. In AL reinfections the haplotype NFD was selected with a prevalence of 10/35 (28.6\%) compared to 29/198 (14.6\%) at baseline $(p=0.05)$, while the prevalence of the haplotype YFD significantly decreased from $27.3 \%$ at baseline to $2.9 \%$ $(p<0.001)$.

\section{Parasite genotypes in relation to day of reinfection and drug concentration}

The correlation between single parasite genotypes and the time of reinfection or patient blood drug concentration was assessed. After ASAQ-FDC treatment, reinfecting parasites harbouring $p f m d r 11246 \mathrm{Y}$ were observed earlier (median, interquartile range; 25.5, 21-35 days) than reinfections with parasites carrying pfmdr1 D1246 (30, 28-37 days, $p=0.03$ ) (Fig. 1a). There was no significant difference in DAQ day 7 concentrations in patients infected with parasites with the respective genotypes. In patients treated with AL there was no significant difference in reinfection day between pfmdr1 N86 and 86Y reinfections. Lumefantrine concentrations (day 7) were significantly higher in patients reinfected with parasites carrying pfmdr1 N86 (median, interquartile range; 0.32, $0.21-0.41 \mathrm{mg} / \mathrm{L}$ ) than in patients with $p f m d r 186 \mathrm{Y}$ reinfections $(0.2,0-0.24 \mathrm{mg} / \mathrm{L}, \mathrm{p}=0.03)$ (Fig. $1 \mathrm{~b}$ ). All reinfections that occurred in patients with day 7 lumefantrine concentrations higher than $0.31 \mathrm{mg} / \mathrm{L}$ were caused by parasites carrying $p f m d r 1$ N86. There were no significant differences associated with $p f c r t 76$ genotypes.

\section{Discussion}

Treatment with ASAQ-FDC and AL were highly effective against $P$. falciparum malaria in this clinical trial. Recrudescences did not have a significantly lower concentration of lumefantrine or DAQ day 7 [9]. Due to the low number of treatment failures, statistical analyses of the recrudescence genotypes were not meaningful. The number of reinfections was found to be high in the AL arm $(30.0 \%)$ and very high in the ASAQ-FDC arm (43.0\%) by day 42 . In the ASAQ-FDC arm selection of the mutant allele $p f m d r 11246 \mathrm{Y}$ and the haplotypes $p f m d r 1 \mathrm{YYY}$ and pfmdr1 NYY in reinfections was demonstrated in Liberia, showing a similar selection as observed in Tanzania [1] and in Mali [2]. The baseline prevalence of $p f m d r 1$ $1246 \mathrm{Y}$ at the time of this trial 2008-2009 was observed to be higher than in other countries in West Africa [38\% including mixed genotype infections compared to 0-25\% in 19 studies (search: years 2000-2014, $\mathrm{n}<40$, from West Africa)] as reported in literature [10]. The high $p f m d r 1$ $1246 \mathrm{Y}$ baseline prevalence may lead to the very high number of reinfections in the ASAQ-FDC arm in this trial, compared to the AL arm. Since $1246 \mathrm{Y}$ was observed to be selected in ASAQ-FDC reinfections, a higher baseline prevalence of the genotype can result in a larger pool of pfmdr1 1246Y carrying parasites that can survive 
Table 1 Number and prevalence of Plasmodium falciparum polymorphisms pre- and post-treatment and post-treatment selection

\begin{tabular}{|c|c|c|c|c|c|c|c|c|c|c|}
\hline \multirow[t]{3}{*}{ Genotype } & \multicolumn{2}{|c|}{ Pre-treatment } & \multicolumn{8}{|c|}{ Post-treatment } \\
\hline & & & \multicolumn{4}{|c|}{ ASAQ-FDC } & \multicolumn{4}{|c|}{$A L$} \\
\hline & & & \multicolumn{2}{|c|}{ Recrudescences } & \multicolumn{2}{|c|}{ Reinfections } & \multicolumn{2}{|c|}{ Recrudescences } & \multicolumn{2}{|c|}{ Reinfections } \\
\hline \multicolumn{11}{|l|}{ pfcrt 76} \\
\hline K & 19 & $6.5 \%$ & 0 & $0.0 \%$ & 2 & $3.3 \%$ & 1 & $16.7 \%$ & $12^{* * *}$ & $31.6 \%$ \\
\hline $\mathrm{K} / \mathrm{T}$ & 7 & $2.4 \%$ & 0 & $0.0 \%$ & 3 & $5.0 \%$ & 0 & $0.0 \%$ & 1 & $2.6 \%$ \\
\hline T & 268 & $91.2 \%$ & 2 & $100.0 \%$ & 55 & $91.7 \%$ & 5 & $83.3 \%$ & 25 & $65.8 \%$ \\
\hline \multicolumn{11}{|l|}{ pfmdr1 86} \\
\hline $\mathrm{N}$ & 90 & $30.6 \%$ & 1 & $50.0 \%$ & 12 & $19.7 \%$ & 4 & $66.7 \%$ & $28^{* * *}$ & $70.0 \%$ \\
\hline$N / Y$ & 47 & $16.0 \%$ & 0 & $0.0 \%$ & 8 & $13.1 \%$ & 1 & $16.7 \%$ & 3 & $7.5 \%$ \\
\hline Y & 157 & $53.4 \%$ & 1 & $50.0 \%$ & 41 & $67.2 \%$ & 1 & $16.7 \%$ & 9 & $22.5 \%$ \\
\hline \multicolumn{11}{|l|}{ pfmdr1 184} \\
\hline Y & 166 & $56.5 \%$ & 1 & $50.0 \%$ & 33 & $55.9 \%$ & 3 & $50.0 \%$ & 24 & $55.8 \%$ \\
\hline Y/F & 24 & $8.2 \%$ & 1 & $50.0 \%$ & 2 & $3.4 \%$ & 1 & $16.7 \%$ & 2 & $4.7 \%$ \\
\hline $\mathrm{F}$ & 104 & $35.4 \%$ & 0 & $0.0 \%$ & 24 & $40.7 \%$ & 2 & $33.3 \%$ & 17 & $39.5 \%$ \\
\hline \multicolumn{11}{|l|}{ pfmdr 11246} \\
\hline D & 181 & $61.6 \%$ & 2 & $66.7 \%$ & 29 & $48.3 \%$ & 5 & $83.3 \%$ & 33 & $76.7 \%$ \\
\hline$D / Y$ & 58 & $19.7 \%$ & 1 & $33.3 \%$ & 7 & $11.7 \%$ & 0 & $0.0 \%$ & 2 & $4.7 \%$ \\
\hline Y & 55 & $18.7 \%$ & 0 & $0.0 \%$ & $24^{* *}$ & $40.0 \%$ & 1 & $16.7 \%$ & 8 & $18.6 \%$ \\
\hline \multicolumn{11}{|l|}{ pfmrp1 876} \\
\hline I & 285 & $96.9 \%$ & 2 & $100.0 \%$ & 59 & $96.7 \%$ & 6 & $100.0 \%$ & 42 & $97.7 \%$ \\
\hline IN & 7 & $2.4 \%$ & 0 & $0.0 \%$ & 1 & $1.6 \%$ & 0 & $0.0 \%$ & 0 & $0.0 \%$ \\
\hline V & 2 & $0.7 \%$ & 0 & $0.0 \%$ & 1 & $1.6 \%$ & 0 & $0.0 \%$ & 1 & $2.3 \%$ \\
\hline \multicolumn{11}{|l|}{ pfmrp1 1466} \\
\hline K & 283 & $96.3 \%$ & 3 & $100.0 \%$ & 60 & $100.0 \%$ & 6 & $100.0 \%$ & 40 & $100.0 \%$ \\
\hline $\mathrm{K} / \mathrm{R}$ & 4 & $1.4 \%$ & 0 & $0.0 \%$ & 0 & $0.0 \%$ & 0 & $0.0 \%$ & 0 & $0.0 \%$ \\
\hline $\mathrm{R}$ & 7 & $2.4 \%$ & 0 & $0.0 \%$ & 0 & $0.0 \%$ & 0 & $0.0 \%$ & 0 & $0.0 \%$ \\
\hline \multicolumn{11}{|c|}{ pfmdr1 $86+184+1246^{a}$} \\
\hline NYD & 55 & $27.8 \%$ & 1 & $100.0 \%$ & 5 & $10.2 \%$ & 2 & $40.0 \%$ & 15 & $42.9 \%$ \\
\hline NYY & 0 & $0 \%$ & 0 & $0 \%$ & $2^{*}$ & $4.1 \%$ & 0 & $0 \%$ & 1 & $2.9 \%$ \\
\hline NFD & 29 & $14.6 \%$ & 0 & $0.0 \%$ & 4 & $8.2 \%$ & 2 & $40.0 \%$ & $10^{*}$ & $28.6 \%$ \\
\hline YFD & 54 & $27.3 \%$ & 0 & $0.0 \%$ & 15 & $30.6 \%$ & 0 & $0.0 \%$ & 1 & $2.9 \%$ \\
\hline YYY & 47 & $23.7 \%$ & 0 & $0.0 \%$ & $20^{*}$ & $40.8 \%$ & 1 & $20.0 \%$ & 5 & $14.3 \%$ \\
\hline Others $^{b}$ & 13 & $6.6 \%$ & 0 & $0.0 \%$ & 3 & $6.1 \%$ & 0 & $0.0 \%$ & 3 & $8.6 \%$ \\
\hline
\end{tabular}

Number and prevalence of polymorphisms selected post-treatment are in italics

${ }^{*} \mathrm{p} \leq 0.05,{ }^{* *} \mathrm{p}<0.001,{ }^{* * *} \mathrm{p}<0.0001$

a Haplotype prevalence excluding mixed genotype infections

b YYD, NFY, YFY

the residual DAQ levels and result in more reinfections. Selection of pfmdr1 $1246 \mathrm{Y}$ was observed mainly in early reinfections, while after day 35 the prevalence of $1246 \mathrm{Y}$ in reinfection was similar to the $1246 \mathrm{Y}$ prevalence before treatment. These results indicate that the concentration of DAQ was high enough to provide a selection pressure up to day 35 . After this time-point all genotypes were able to survive the residual drug level and cause reinfection and there was no longer a protective or selective effect of the drug. This idea needs to be further elaborated by studying further efficacy trials with ASAQ and AL in West Africa. The 1246Y genotype has also been associated with ASAQFDC treatment failures [4]. Despite the high prevalence of $1246 \mathrm{Y}$, few treatment failures were observed in this study, probably due to the high efficacy of the artesunate compound in the combination. Artemisinin resistance is defined as delayed parasite clearance after treatment with an artemisinin or ACT [11]. In this study a low proportion 

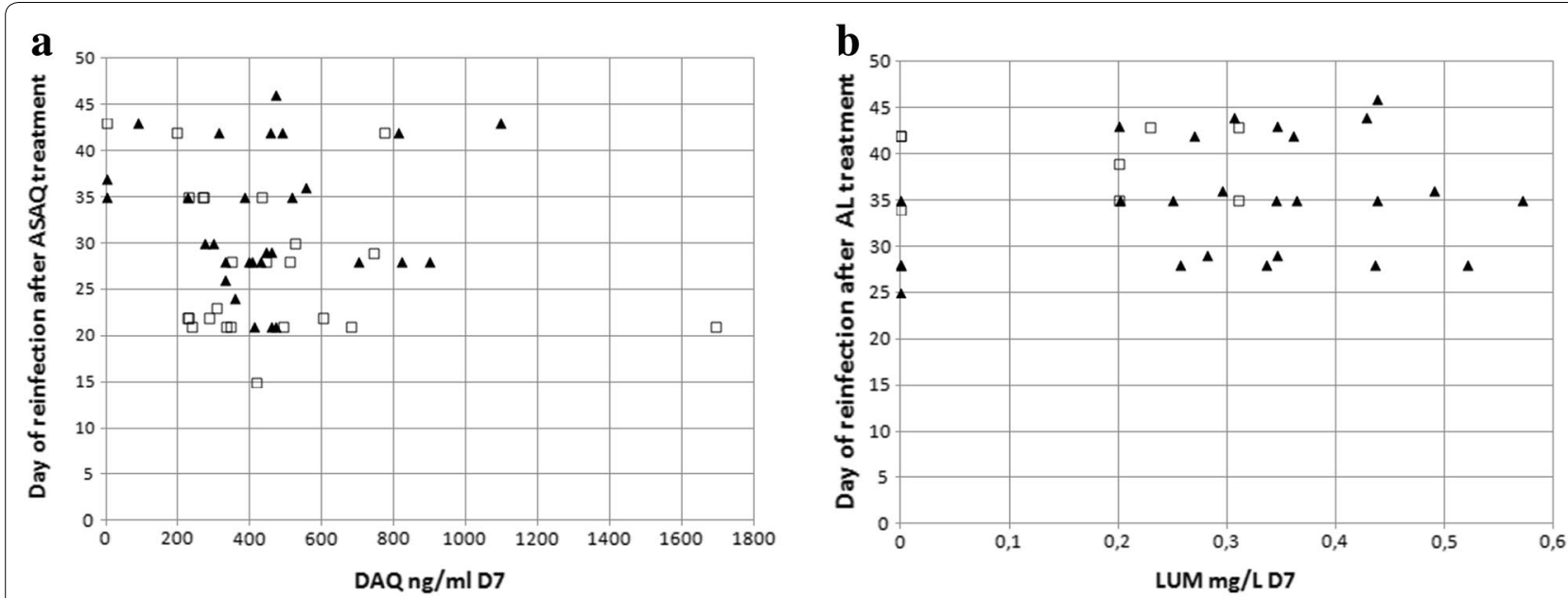

Fig. 1 Plot of reinfection genotypes by day and patient blood drug concentration day 7. Each data point represents the P. falciparum genotype of a reinfection. a Genotype of reinfections after ASAQ-FDC treatment and desethylamodiaquine (DAQ) concentration. Filled triangles represent reinfections harbouring the pfmdr1 D1246 genotype and open squares represent pfmdr1 1246Y reinfections. b Genotype of reinfections after AL treatment and lumefantrine (LUM) concentration. Filled triangles represent reinfections harbouring the pfmdr1 N86 genotype and open squares represent pfmdr1 86Y reinfections

of patients were parasite positive on day 3 [9], indicating high efficacy of the artesunate compound.

In the AL arm, the wild-type alleles $p f c r t \mathrm{~K} 76, p f m d r 1$ N86 and the $p f m d r 1$ haplotype NFD were selected in reinfecting parasites, by the drug pressure of residual levels of lumefantrine. Parasites harbouring pfmdr1 N86 was not observed to reinfect significantly earlier, as has been suggested in previous studies $[4,6]$. However it was demonstrated that parasites carrying pfmdr1 N86 could cause reinfection in patients with higher lumefantrine concentrations, than parasites carrying pfmdr1 86Y. Patients with low lumefantrine levels could be reinfected with both N86 and 86Y alleles, while parasites with pfmdr1 N86 could withstand intermediate drug concentrations. At high lumefantrine concentrations both genotypes would be killed and no reinfection could occur. This idea is supported by the observation that patients with reinfections had lower lumefantrine levels [9]. This is in agreement with an important study suggesting that reinfecting parasites with $p f m d r 1 \mathrm{~N} 86$ as well as the $p f m d r 1 \mathrm{~N} 86 / 184 \mathrm{~F} /$ D1246 haplotype can withstand higher lumefantrine concentrations based on day 7 data [12]. Both individual drug levels and the day of reinfection are key determinants for selection of reinfections and needs to be taken into account if available. This is especially important for AL-treated patients, in which the total lumefantrine dose received can vary significantly [13]. In studies from East Africa $p f m d r 1184 \mathrm{~F}$ is often selected in reinfections after AL treatment, which was not observed in this study. This could be due to the high baseline prevalence of $p f m d r 1$ NYD haplotype in this and a study in Benin [3], where pfmdr1 N86 could be the main driving force for lumefantrine resistance, independently of the $p f m d r 1184$ genotype.

The longer elimination half-life of the partner drug is a double-edged sword since although it can provide some post-prophylactic protection of individual patients, selection of reinfections can result in a more resistant parasite population over time. In high transmission areas, in Tanzania and Uganda, consistent treatment with AL overtime has probably resulted in significant increases in the prevalence of genotypes associated with AL treatment failure and reinfection $[14,15]$. However, temporal changes of ASAQ resistance markers have not been observed after consistent use of AS-AQ in Zanzibar [16].

\section{Conclusions}

In this first study investigating pfcrt and pfmdrl polymorphisms after the implementation of ACT in Liberia, selection of molecular markers in AL and ASAQ-FDC reinfections and high pfmdr1 $1246 \mathrm{Y}$ baseline prevalence was demonstrated. The observation that parasites carrying pfmdr1 N86 can reinfect patients with higher lumefantrine concentrations highlights the importance of studying drug levels after AL treatment, as there could be large inter-individual dose variations. It is important to further investigate variables governing selection of reinfections in individual studies and as well as over time, as they can be responsible for the step from selection of a reinfection to full resistance and treatment failures. To evaluate and advise the current treatment policy it is essential to monitor temporal changes in molecular resistance markers of treatments used in Liberia in conjunction with conventional efficacy testing. 


\section{Authors' contributions}

SDO participated in the genetic analysis, performed the statistical analysis and drafted the paper, OMA participated in the genetic analysis, BS participated in the clinical trial, VJ participated in the drug concentration analysis, JJ participated in the clinical trial, YZ participated in the clinical trial, $\mathrm{PH}$ participated in the drug concentration analysis, EA participated in the conception of the clinical trial, JK participated in the conception of the clinical trial, PG participated in the conception of the clinical trial and the study, JLB participated in the conception of the study and participated in the coordination and the design of the study, SH participated in the conception of the study and participated in the coordination and the design of the study. All authors read and approved the final manuscript.

\section{Author details}

${ }^{1}$ Institut de Médecine et d'Epidémiologie Appliquée, Bichat-C. Bernard Hospital, Paris, France. ${ }^{2}$ WorldWide Antimalarial Resistance Network, Oxford, UK. ${ }^{3}$ Johnson C. Smith University, Charlotte, NC 28216, USA. ${ }^{4}$ Bernhard Nocht Institute for Tropical Medicine, 20359 Hamburg, Germany. ${ }^{5}$ Epicentre, 75012 Paris, France. ${ }^{6}$ INSERM U1129, Service de Pharmacologie, Hôpital Européen Georges Pompidou, Université Paris Descartes, 75015 Paris, France. ${ }^{7}$ National Malaria Control Programme, Ministry of Health and Social Welfare, Monrovia, Liberia. ${ }^{8}$ AP-HP, Saint-Louis Hospital Biochemistry Laboratory, Paris, France. ${ }^{9}$ Nuffield Department of Clinical Medicine, Centre for Tropical Medicine and Global Health, University of Oxford, Oxford, UK. ${ }^{10}$ Drugs for Neglected Diseases Initiative, Geneva, Switzerland. ${ }^{11}$ IRD UMR216, ParisDescartes University, Paris, France. ${ }^{12}$ Parasitology Laboratory-French National Malaria Reference Centre, AP-HP, Bichat-C. Bernard Hospital, Paris, France.

\section{Acknowledgements}

We thank the children participating in this trial and their guardians.

\section{Competing interests}

Dr. Kiechel was the DNDi Project manager for developing the fixed dose combination of artesunate-amodiaquine used in this study-ASAQ Winthrop ${ }^{\circledR}$, Sanofi-Aventis.

\section{Availability of data and material}

Data from this study is deposited in the repository of the World Wide Antimalarial Resistance Network, which is registered with the Registry of Research Data Repositories (re3data.org). Data can be requested through the WWARN website (http://www.wwarn.org/working-together/sharing-data/data-available-research-community) and approved requestors will be provided with a copy of the original dataset used for the publication.

\section{Ethics approval and consent to participate}

The studies were approved by the Liberian Institute for Biomedical Research (LIBR) Ethics Committee, the Ministry of Health and Social Welfare, Monrovia, Liberia, and the Comité de Protection des Personnes (CPP) Ile de France XI (Saint Germain en Laye), France. All participants or responsible caretakers signed informed consent.

\section{Funding}

This study was funded by Drugs for Neglected Diseases Initiative (DNDi).

\section{Meetings}

Part of this work was presented at the American Society of Tropical Medicine and Hygiene 60th Annual Meeting 4-8th December, 2011, Philadelphia, PA, USA (poster LB-2171).

Received: 26 March 2016 Accepted: 26 August 2016

Published online: 05 September 2016

\section{References}

1. Holmgren G, Hamrin J, Svard J, Mårtensson A, Gil JP, Björkman A. Selection of pfmdr 1 mutations after amodiaquine monotherapy and amodiaquine plus artemisinin combination therapy in East Africa. Infect Genet Evol. 2007;7:562-9.
2. Djimdé AA, Fofana B, Sagara I, Sidibe B, Toure S, Dembele D, et al. Efficacy, safety, and selection of molecular markers of drug resistance by two ACTs in Mali. Am J Trop Med Hyg. 2008;78:455-61.

3. Dahlström S, Aubouy A, Maïga-Ascofaré O, Faucher JF, Wakpo A, Ezinmègnon $\mathrm{S}$, et al. Plasmodium falciparum polymorphisms associated with ex vivo drug susceptibility and clinical effectiveness of artemisininbased combination therapies in Benin. Antimicrob Agents Chemother. 2014;58:1-10.

4. Venkatesan M, Gadalla NB, Stepniewska K, Dahal P, Nsanzabana C, Moriera C, et al. Polymorphisms in Plasmodium falciparum chloroquine resistance transporter and multidrug resistance 1 genes: parasite risk factors that affect treatment outcomes for P. falciparum malaria after artemether-lumefantrine and artesunate-amodiaquine. Am J Trop Med Hyg. 2014;91:833-43.

5. Dokomajilar C, Nsobya SL, Greenhouse B, Rosenthal PJ, Dorsey G. Selection of Plasmodium falciparum pfmdr1 alleles following therapy with artemether-lumefantrine in an area of Uganda where malaria is highly endemic. Antimicrob Agents Chemother. 2006:50:1893-5.

6. Sisowath C, Ferreira PE, Bustamante LY, Dahlström S, Mårtensson A, Björkman A, et al. The role of pfmdr 1 in Plasmodium falciparum tolerance to artemether-lumefantrine in Africa. Trop Med Int Health. 2007;12:736-42.

7. Jovel IT, Ferreira PE, Veiga MI, Malmberg M, Mårtensson A, Kaneko A, et al. Single nucleotide polymorphisms in Plasmodium falciparum $V$ type $\mathrm{H}(+)$ pyrophosphatase gene (pfvp2) and their associations with pfcrt and pfmdr1 polymorphisms. Infect Genet Evol. 2014;24:111-5.

8. Checchi F, Durand R, Balkan S, Vonhm BT, Kollie JZ, Biberson P, et al. High Plasmodium falciparum resistance to chloroquine and sulfadoxinepyrimethamine in Harper, Liberia: results in vivo and analysis of point mutations. Trans R Soc Trop Med Hyg. 2002;96:664-9.

9. Schramm B, Valeh P, Baudin E, Mazinda CS, Smith R, Pinoges L, et al. Efficacy of artesunate-amodiaquine and artemether-lumefantrine fixed-dose combinations for the treatment of uncomplicated Plasmodium falciparum malaria among children aged six to 59 months in Nimba County, Liberia: an open-label randomized non-inferiority trial. Malar J. 2013;12:251.

10. Molecular Surveyor pfmdr1 and pfcrt. WorldWide Antimalarial Resistance Network (WWARN). http://www.wwarn.org/tracking-resistance/molecular-surveyor-pfmdr1-pfcrt. Accessed 1 Feb 2015

11. WHO: Update on artemisinin and ACT resistance. Geneva: World Health Organization; April 2016.

12. Malmberg M, Ferreira PE, Tarning J, Ursing J, Ngasala B, Björkman A, et al. Plasmodium falciparum drug resistance phenotype as assessed by patient antimalarial drug levels and its association with pfmdr 1 polymorphisms. J Infect Dis. 2013:207:842-7.

13. Worldwide Antimalarial Resistance Network (WWARN) AL Dose Impact Study Group. The effect of dose on the antimalarial efficacy of artemether-lumefantrine: a systematic review and pooled analysis of individual patient data. Lancet Infect Dis. 2015;15(6):692-702.

14. Malmberg M, Ngasala B, Ferreira PE, Larsson E, Jovel I, Hjalmarsson A, et al. Temporal trends of molecular markers associated with artemetherlumefantrine tolerance/resistance in Bagamoyo district, Tanzania. Malar J. 2013;12:103.

15. Mbogo GW, Nankoberanyi S, Tukwasibwe S, Baliraine FN, Nsobya SL, Conrad MD, et al. Temporal changes in prevalence of molecular markers mediating antimalarial drug resistance in a high malaria transmission setting in Uganda. Am J Trop Med Hyg. 2014;91:54-61.

16. Fröberg G, Jörnhagen L, Morris U, Shakely D, Msellem MI, Gil JP, et al. Decreased prevalence of Plasmodium falciparum resistance markers to amodiaquine despite its wide scale use as ACT partner drug in Zanzibar. Malar J. 2012;11:321. 